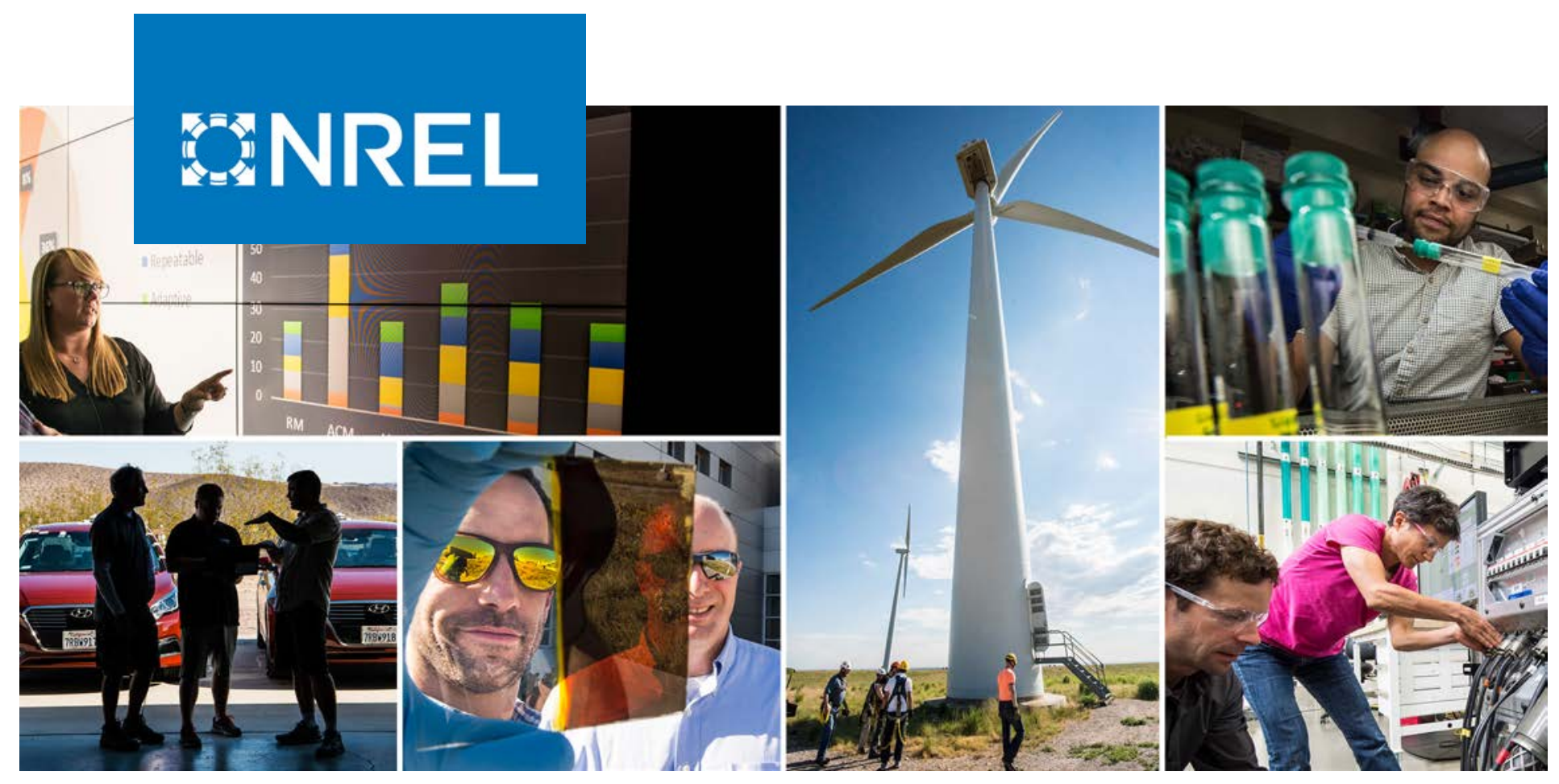

\title{
Feasibility Analysis of Taxi Fleet Electrification using 4.9 Million Miles of Real-World Driving Data
}

\section{Preprint}

Matthew Moniot, Clement Rames, and Erin Burrell

National Renewable Energy Laboratory

Presented at SAE WCX World Congress Experience

Detroit, Michigan

April 9-11, 2019

NREL is a national laboratory of the U.S. Department of Energy

Office of Energy Efficiency \& Renewable Energy

Operated by the Alliance for Sustainable Energy, LLC

This report is available at no cost from the National Renewable Energy Laboratory (NREL) at www.nrel.gov/publications.

\section{Conference Paper}

NREL/CP-5400-72944

June 2019 


\section{ENREL}

\section{Feasibility Analysis of Taxi Fleet Electrification using 4.9 Million Miles of Real-World Driving Data}

\section{Preprint}

Matthew Moniot, Clement Rames, and Erin Burrell

National Renewable Energy Laboratory

\section{Suggested Citation}

Moniot, Matthew, Clement Rames, and Erin Burrell. 2019. Feasibility Analysis of Taxi

Fleet Electrification using 4.9 Million Miles of Real-World Driving Data: Preprint. Golden,

CO: National Renewable Energy Laboratory. NREL/CP-5400-72944.

https://www.nrel.gov/docs/fy19osti/72944.pdf.

NREL is a national laboratory of the U.S. Department of Energy Office of Energy Efficiency \& Renewable Energy Operated by the Alliance for Sustainable Energy, LLC

This report is available at no cost from the National Renewable Energy Laboratory (NREL) at www.nrel.gov/publications.

Contract No. DE-AC36-08GO28308
Conference Paper

NREL/CP-5400-72944

May 2019

National Renewable Energy Laboratory 15013 Denver West Parkway Golden, CO 80401

303-275-3000 • www.nrel.gov 


\section{NOTICE}

This work was authored by the National Renewable Energy Laboratory, operated by Alliance for Sustainable Energy, LLC, for the U.S. Department of Energy (DOE) under Contract No. DE-AC36-08GO28308. Funding provided by the U.S. Department of Energy Office of Energy Efficiency and Renewable Energy Vehicle Technologies Office. The views expressed herein do not necessarily represent the views of the DOE or the U.S. Government. The U.S. Government retains and the publisher, by accepting the article for publication, acknowledges that the U.S. Government retains a nonexclusive, paid-up, irrevocable, worldwide license to publish or reproduce the published form of this work, or allow others to do so, for U.S. Government purposes.

This report is available at no cost from the National Renewable Energy Laboratory (NREL) at www.nrel.gov/publications.

U.S. Department of Energy (DOE) reports produced after 1991 and a growing number of pre-1991 documents are available free via www.OSTI.gov.

Cover Photos by Dennis Schroeder: (clockwise, left to right) NREL 51934, NREL 45897, NREL 42160, NREL 45891, NREL 48097, NREL 46526.

NREL prints on paper that contains recycled content. 


\title{
Feasibility Analysis of Taxi Fleet Electrification using 4.9 Million Miles of Real- World Driving Data
}

\author{
Matthew Moniot, Clement Rames, Erin Burrell \\ National Renewable Energy Laboratory
}

\begin{abstract}
Ride hailing activity is rapidly increasing, largely due to the growth of transportation network companies such as Uber and Lyft. However, traditional taxi companies continue to represent an important mobility option for travelers. Columbus Yellow Cab, a taxi company in Columbus, Ohio, offers traditional line-of-sight hailing as well as digital hailing through a mobile app. Data from Columbus Yellow Cab was provided to the National Renewable Energy Laboratory to analyze the potential for taxi electrification. Columbus Yellow Cab data contained information describing both global positioning system trajectories and taxi meter information. The data spanned a period of 13 months, containing approximately 70 million global system positioning system points, 840 thousand trips, and 170 unique vehicles. A variety of scenarios were evaluated using Columbus Yellow Cab data and the Electric Vehicle Infrastructure Projection Tool (EVI-Pro) to understand challenges and opportunities associated with operating an electrified taxi fleet. Two main factorsaccess to home charging and vehicle specifications - are shown to be major variables affecting successful electric fleet operation. The analysis indicates that $95.7 \%$ of taxi travel days can be successfully completed by a 250-mile-range electric vehicle assuming access to overnight and public charging infrastructure. However, when no overnight access is available to fleet vehicles, only $39.9 \%$ of taxi travel days are possible with 250-mile range electric vehicles. An additional scenario, reducing the vehicle range from 250 miles to 100 miles (while controlling for infrastructure access and permitting overnight charging) resulted in only $34.4 \%$ of taxi travel days being completed.
\end{abstract}

\section{Introduction}

As various industries are curtailing their emissions due to increased energy efficiency measures, the amount of greenhouse gas (GHG) emissions originating from the transportation sector continues to rise. The transportation sector recently passed electricity generation as the largest source of GHG emissions by sector in the United States in 2016, totaling 28\% of all emissions. Emissions from light-duty vehicles alone represented $60 \%$ of all GHG emissions within the transportation category [1]. Cities and municipalities interested in reducing their GHG levels are increasingly passing measures specifically related to light-duty vehicles, most notably through electric vehicle purchase incentives and charging infrastructure investments [2][3][4]. The rise in transportation-related emissions has occurred alongside changes in mobility behavior due to more available mode options, most notably transportation network companies (TNCs). TNC vehicles are shown to have significantly higher utilization and annual miles driven. For instance, a publicly released dataset from the ride-hailing company RideAustin revealed that full-time TNC vehicles were being driven at an average distance of 29,000 miles per year, far higher than the national average of 11,488 miles per year in 2016 for personally owned vehicles [5][6]. The rise of TNCs, simultaneous with the increasing share of GHG emissions originating from the transportation sector, has led some legislatures to begin exploring fuel economy and electrification requirements specifically for TNC vehicles. Most notably, the State of California recently approved legislation establishing future goalsetting for electrification of TNC vehicles [7].

The rapid growth of TNCs has disrupted traditional taxi services. For instance, data from the Los Angeles Department of Transportation show taxi ridership falling by nearly $30 \%$ between 2012 and 2015 within the city [8]. However, these services are quickly adapting. Many fleets are beginning to offer more sophisticated means of hailing trips (through websites, mobile phone apps, etc.), and changing regulations are reducing some barriers that inhibit taxi services from more effectively competing with TNC companies [9] [10]. Given the rising interest of legislators regulating for-hire lightduty vehicles and the lower cost of operation of electric vehicles, there is considerable enthusiasm surrounding the question of electrifying TNCs and taxi fleets.

One such fleet, Columbus Yellow Cab (CYC), operates approximately 170 gasoline and hybrid electric vehicles within the city of Columbus Ohio. NREL researchers collaborated with CYC to explore the feasibility of transitioning its fleet of gasoline vehicles to electric vehicles given the growth of commercial ride hailing and large electrification potential. To better understand this question, CYC shared data with the National Renewable Energy Laboratory (NREL) for vehicle and infrastructure analysis. The data contain over 70 million global positioning system (GPS) points from 170 unique vehicles, describing 840 thousand trips over a period of 13 months. First, a detailed data exploration is presented, highlighting key similarities and differences between taxi and TNC utilization. Next, feasibility of electrification is explored using an infrastructure projection tool that highlights vehicle-level and infrastructure-level requirements necessary to support fleet operations. Finally, results from sensitivity analysis of vehicle electric range and infrastructure availability are presented.

\section{Columbus Yellow Cab Data}

The data provided to NREL describing CYC are unique in scope and vocation. This section starts by introducing the size and geographic extent of the dataset, relevant fields, and summary statistics describing fleet operation. The latter portions of this section compare CYC fleet vehicle behavior to personally owned vehicles and vehicles operated by full-time RideAustin drivers. These comparisons are introduced to understand how vehicle utilization may vary for taxi 
vehicles and to identify any challenges associated with fleet electrification.

\section{Data Summary and Processing}

CYC supplied 13 months (April 2017-April 2018) of GPS data describing fleet operation for all of its vehicles. The supplied data consisted of 70 million unique GPS data points describing longitude and latitude, timestamp, and a vehicle identifier. An additional field, the taxi meter, distinguished a paid trip with a passenger(s) versus vehicle travel with no passenger, or empty miles. The geographic extent of the GPS data is visualized as a heatmap in Figure 1, with more frequent travel locations shown in a brighter color. Fleet travel behavior is intuitive - trips primarily take place in the urban center and on interstates, with fewer trips traveling on arterial roadways outside of the city.

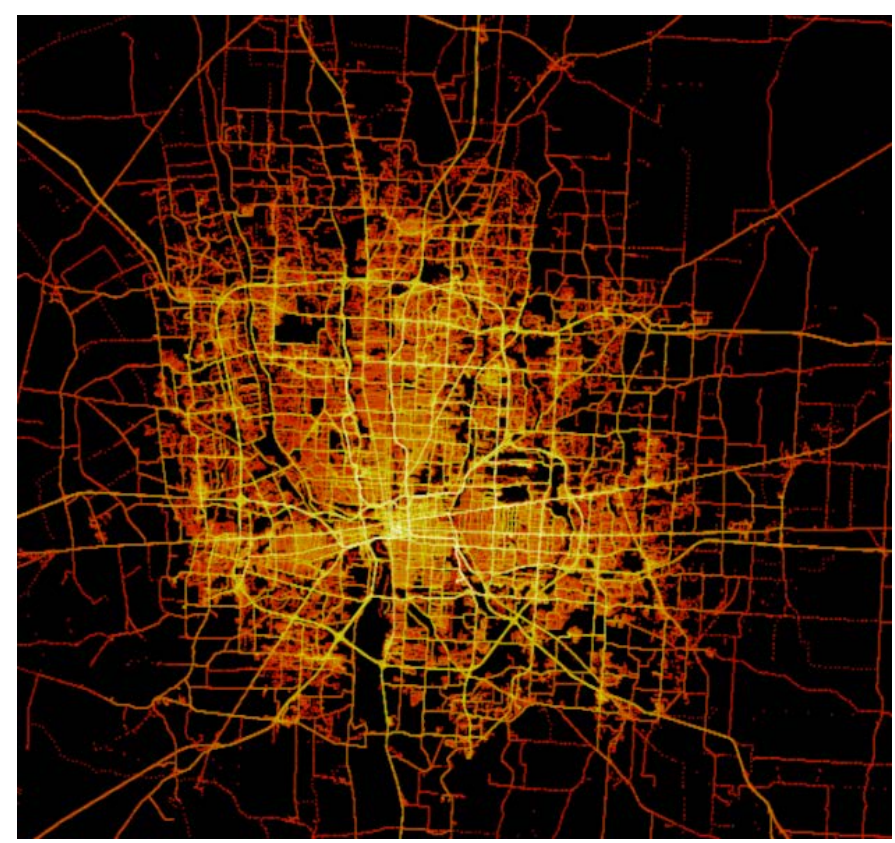

Figure 1. Heatmap describing geographic extent of the 70 million unique GPS points.

The GPS data were then segmented into over 840 thousand trips, based on any changes in the meter status and calculations involving the vehicle speed. Park events, or dwells, were also identified to understand any opportunities for charge events. Trips without a passenger will be referred to as deadheading-trips. Finally, trip segmentation also required categorizing locations of trip origins and destination at the residence of a CYC driver, or a public location. Describing location type at the end of each trip is necessary for understanding access to potential charging opportunities. Additional information regarding infrastructure modeling is located in the PEV Infrastructure Modeling section.

The number of active vehicles varied throughout the 13-month timespan with an average of 146 unique fleet vehicles being utilized per month out of the total 170 unique vehicles present in the dataset. Average annual operation amounted to 280 days of use per year per vehicle and 154 miles per day driven. In total, 35,112 unique vehicledays were present within the dataset. These vehicle-days comprise one or more trips taking place during a particular day by a particular vehicle. A vehicle-day describes the 24-hour sequence of driving and park events, summarizing the amount of driving completed and the number of park events available for potential vehicle charging.
CYC vehicle use varies considerably between weekdays and weekends. Most notably, analysis of the provided dataset revealed that fewer vehicles operated on weekends and that these same vehicles traveled fewer miles. Figure 2 describes the cumulative distribution of the number daily miles traveled (dVMT) of fleet vehicles with separate traces by day of week. Inspection of the cumulative dVMT distribution reveals that $10 \%$ of fleet vehicle-days during a weekday contain over 250 miles of travel compared to only 200 miles of dVMT for vehicle-days occurring on weekends. Beyond illustrating that vehicles travel less on Saturday and Sunday, Figure 2 also shows there is minimal variance between travel on a particular day during the week or a particular day during the weekend.

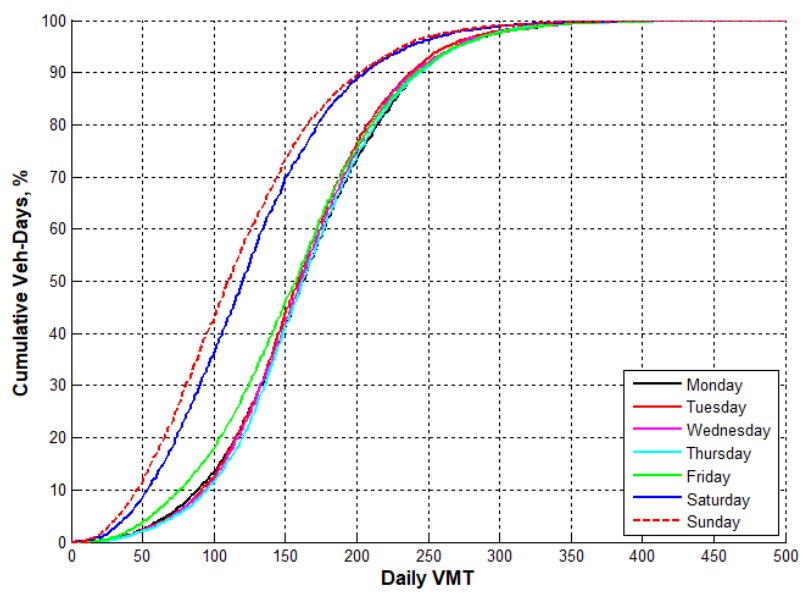

Figure 2. Cumulative CYC dVMT distributions separated by day of week.

Variation by day of week is also found when analyzing when trips take place by time of day. Figure 3 describes the relative frequency of trip start times by hour of day with unique traces for each day of the week. Trip frequency by time of day is consistent across weekdays, with a mid-day peak and minimal travel during overnight hours, except for Friday, with a larger trip frequency toward the end of the day. Larger shares of overnight travel can be also found in Saturday evening and Sunday morning.

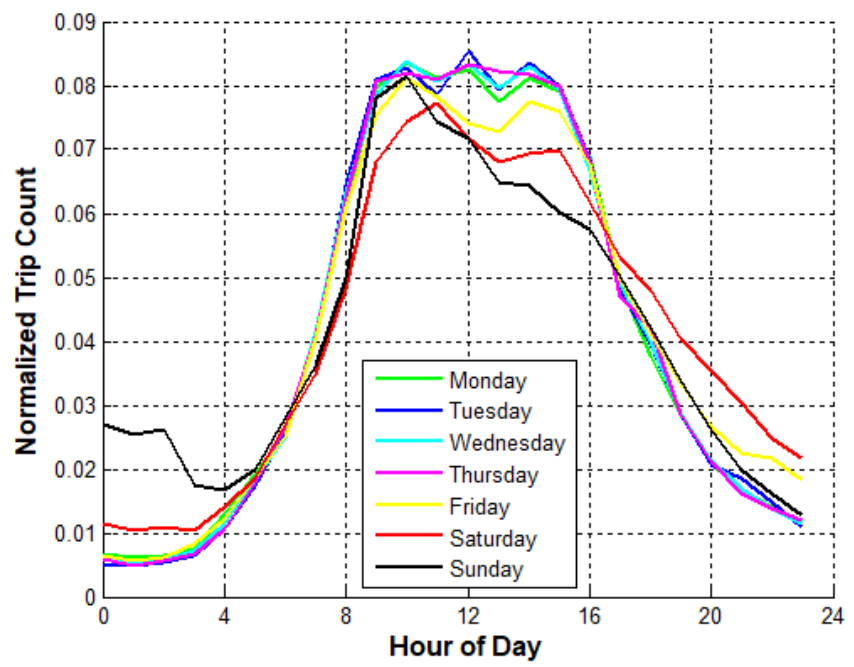

Figure 3. CYC trip frequency by time of day and separated by day of week.

Together, Figure 2 and Figure 3 show that the day of the week strongly influences fleet operation, and weekdays may require the largest amount of infrastructure support due to the larger dVMT distributions. Additionally, the minimal variance in travel behavior at the daily level within the categories of weekday and weekend 
supports characterizing vehicle-days categorically as either weekday or weekend.

\section{Comparison to Personal Travel}

Taxi vehicles have considerably different activity patterns than vehicles utilized for personal travel. Analysis of the CYC data facilitated a unique opportunity to compare fleet vehicle operation and personal vehicle operation while controlling for geographic area, given prior research performed by NREL that focused on the city of Columbus [11]. A prior study researching the necessary infrastructure requirements for Columbus, Ohio, performed similar GPS processing of data provided by INRIX, which described passenger travel with personal vehicles. Specific information describing the processing for personal travel can be found in the Columbus infrastructure report [11].

The most striking difference between taxi vehicle operation and personal vehicle operation is larger utilization of taxi vehicles. While a personal vehicle accommodates the needs of few travelers with a short number of trips and longer dwell times, taxi vehicles travel longer periods of time, are driven over greater distances, and typically experience long dwells only overnight. Figure 4 illustrates the dVMT distribution for CYC fleet vehicles versus primarily personally owned vehicles contained within the INRIX dataset. Inspection shows that $20 \%$ of CYC fleet vehicle-days contain more than 200 miles of driving. Meanwhile, less than $1 \%$ of vehicle-days within the INRIX dataset exceeded 200 miles of driving in a day.

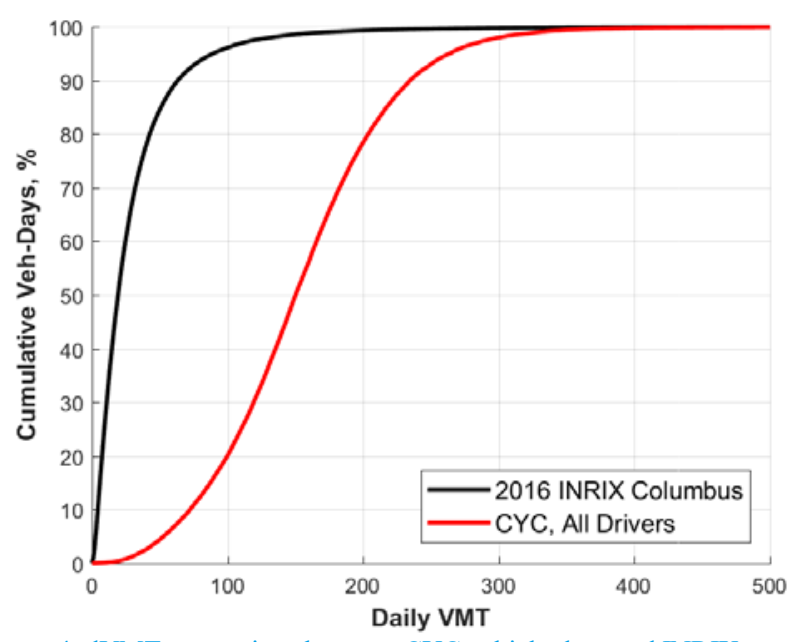

Figure 4. dVMT comparison between CYC vehicle-days and INRIX Columbus vehicle-days.

In addition to analyzing the $\mathrm{dVMT}$ distributions for CYC and personally owned vehicles, additional comparisons are shown in Figure 5 describing trip counts by time of day. Note that the CYC curve represents an aggregation of the seven traces shown in Figure 3. Trip frequencies from the INRIX Columbus dataset vary considerably by time of day, likely influenced by travel to and from workplaces. A morning peak occurs as travelers leave home, followed by a larger afternoon peak coinciding with the end of a typical workday. Finally, overnight trips within the INRIX Columbus dataset are shown to be less frequent than overnight trips within the CYC dataset. This finding supports the notion that travelers are disproportionately likely to consider using a taxi for late night trips versus their own personally owned vehicles.

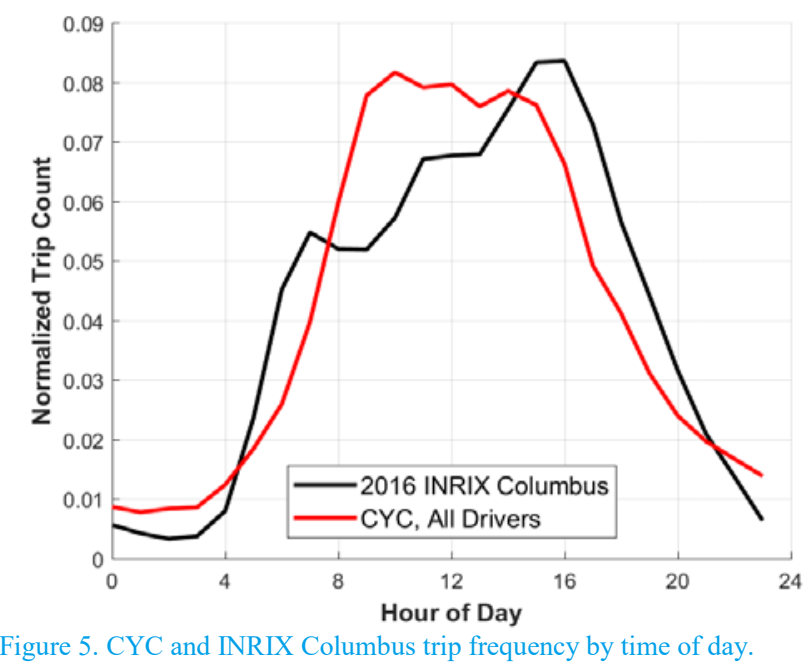

\section{Comparison to TNC Travel}

Taxi fleets and TNCs represent distinct approaches to providing customers with transportation solutions; taxi fleets are primarily composed of full-time drivers using fleet vehicles while TNCs tend to be composed of drivers using their personal vehicles with varying levels of time commitment. However, as fleets incorporate ridehailing functionality through mobile applications, the differences between these services may begin to wane. Additional comparative analysis between the CYC data and publicly available TNC data was performed to understand the similarities and differences of vehicle utilization for these services.

Ideally, CYC operation would be compared against data describing TNC travel also taking place within Columbus, Ohio. Unfortunately, no such dataset is publicly available for researchers from TNCs operating within the region. Instead, data from the nonprofit ridesharing company RideAustin were used to compare against the CYC data. The open-source RideAustin dataset contains 1.49 million trips driven by approximately 5,000 unique drivers during a 10month period between June 2016 and April 2017. Dataset information contains important trip parameters including trip distance, pickup / drop-off coordinates, and start / end times. Similar analysis described in the Data Summary and Processing section was performed on the RideAustin dataset and may be found in [5]; the remainder of this section compares the processed CYC data to the processed RideAustin data.

CYC fleet vehicle operation was found to be similar to full-time RideAustin drivers, defined as those driving more than 35 hours/week ( $11 \%$ of drivers). Recall that CYC vehicles were shown to be driven an average of 280 days per year and 154 miles per day. When evaluated annually, vehicles were utilized at an average annualized vehicle miles traveled of approximately 40,000 miles. Analysis in [5] reveals that full-time RideAustin drivers also travel approximately 280 days per year, but with a smaller average daily vehicle miles traveled of only 88 miles. Figure 6 illustrates the differences in miles traveled per day for all CYC vehicles versus RideAustin vehicles driven by full-time drivers. 


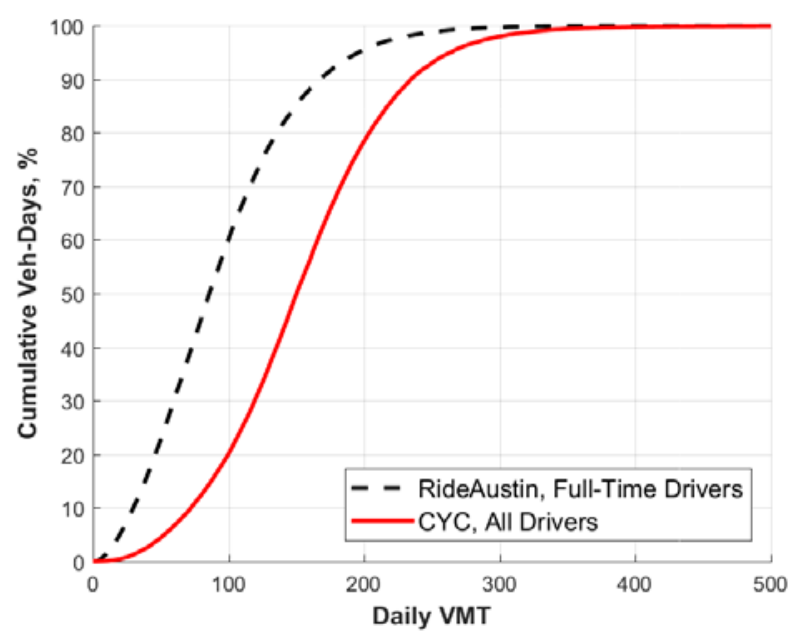

Figure 6. dVMT comparison between CYC vehicle-days and RideAustin vehicle-days.

Time-of-day comparisons - similar to those shown in Figure 5 for personal travel - are also performed for CYC data versus RideAustin Data. Traces are shown for both weekdays and weekends due to the large variance between the day of week and commercial fleet activity. Full-time RideAustin drivers are shown to prioritize overnight hours more than CYC drivers, even on weekdays. This may be partly explained by the flexibility offered by TNC services to work on demand with personal vehicles and the opportunity for increased pricing during hours of increased demand. Prioritization by RideAustin drivers for trips during overnight hours is even more prevalent on weekends, with nearly $53.6 \%$ of trips taking place between the hours of $8 \mathrm{PM}$ and $4 \mathrm{AM}$ as compared to $20.2 \%$ of CYC trips during the same timespan on weekends.

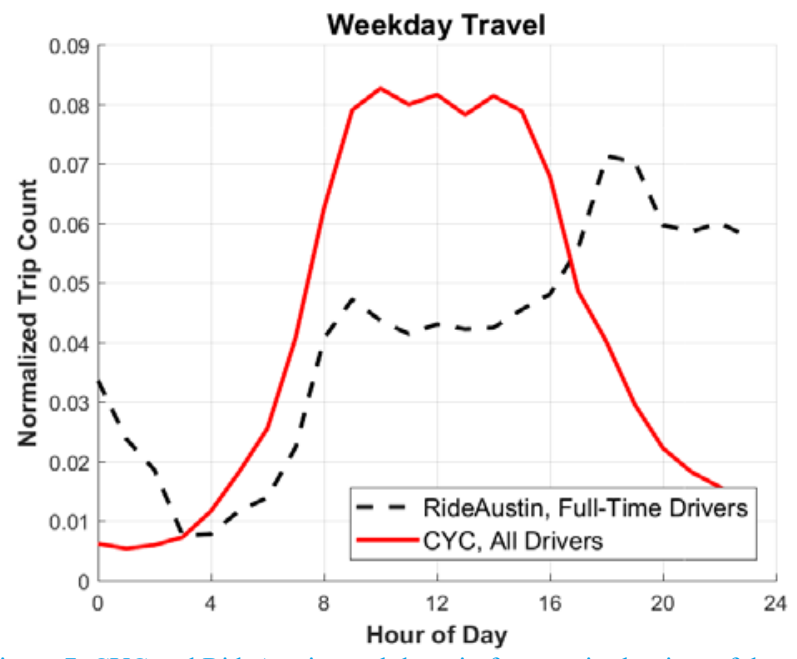

Figure 7. CYC and RideAustin weekday trip frequencies by time of day.

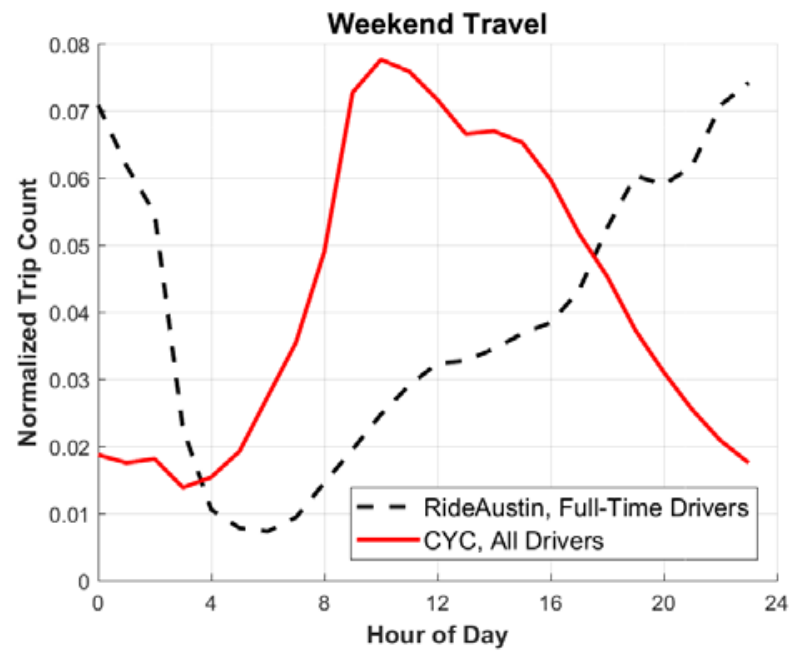

Figure 8. CYC and RideAustin weekend trip frequency by time of day.

[5] also shows that RideAustin vehicles travel more on weekends, while CYC vehicles tend to drive less on weekends. Specifically, the average full-time RideAustin driver travels 81.5 miles per weekday and 96.7 miles per day on weekends, compared to CYC travel of 164 miles per weekday and 123 miles per day on weekends.

The final comparison explored between the CYC and RideAustin datasets evaluates the amount of passenger miles versus empty miles. Analysis of trip information comparing passenger-miles to total miles driven reveals that approximately $54 \%$ of the miles accumulated by CYC vehicles take place with a passenger in the vehicle compared to $46 \%$ of miles driven without a passenger (empty miles). Similar percentages of miles traveled are found in the RideAustin dataset: $51 \%$ of miles traveled are passenger-miles versus the $49 \%$ share of empty-miles.

\section{Ride Austin}

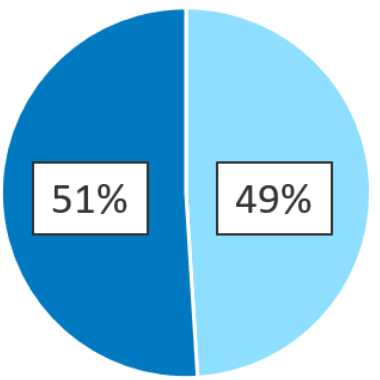

\section{Passenger miles}

\section{Empty miles}

Figure 9. Breakdown of miles driven with and without passengers for CYC vehicles and RideAustin vehicles.

\section{Plug-In Electric Vehicle (PEV) Infrastructure Modeling}

\section{Modeling Overview}

NREL developed the Electric Vehicle Infrastructure Projection Tool (EVI-Pro) in partnership with the California Energy Commission to estimate regional requirements for charging infrastructure to support increased adoption of plug-in electric vehicles (PEVs) $[12,13]$. The 
model aims to anticipate spatially and temporally resolved electric vehicle charging demand while capturing variations with respect to residents of single-unit dwellings and multi-unit dwellings, weekday/ weekend travel behavior, and regional differences in travel behavior and vehicle adoption. Although a capability of the tool, the distinctions for residence type and variances in vehicle adoption are not considered in this study. A graphical representation of the input/output relationships in EVI-Pro is shown in Figure 10.

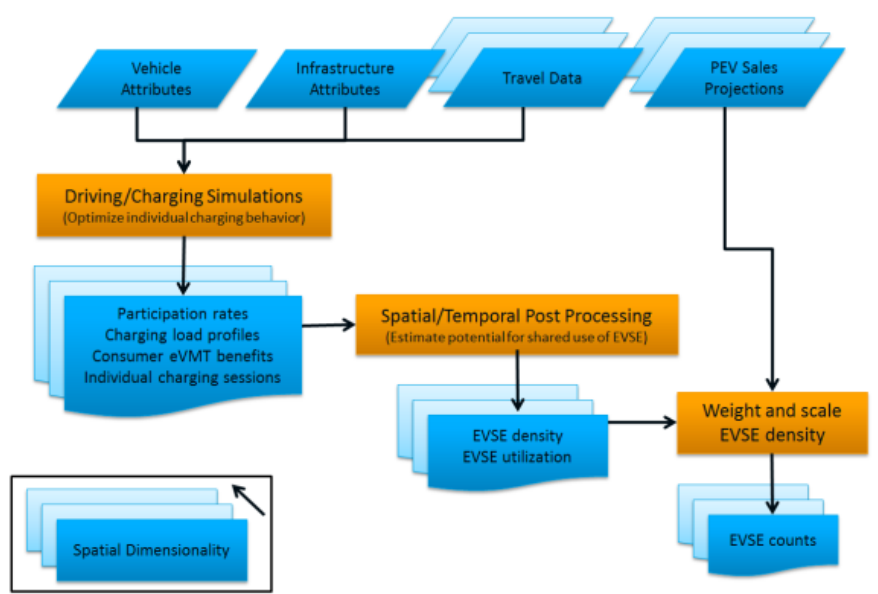

Figure 10. Graphical representation of inputs/outputs and data flow in EVIPro.

EVI-Pro's charging behavior emulation assumes that fleet vehicles aim to complete all their existing travel electrically while minimizing operating cost. Several charging scenarios are simulated for each driver. To identify the optimal charging scenario, individual travel days from the CYC travel dataset (originally completed using a conventional gasoline or hybrid vehicle) are simulated in the model under different assumptions for charging infrastructure availability. EVI-Pro repeats this charge behavior selection routine for all travel days in the study and for all vehicle types under consideration. The modeled PEV fleet consists of entirely of battery electric vehicles with a range of 250 miles (BEV250) and a maximum charging power of $50 \mathrm{~kW}$. The chosen vehicle attributes were selected to be consistent with BEVs that are anticipated to be produced in high quantities (Chevy Bolt, Tesla Model 3). An additional scenario is evaluated assuming fleet vehicles having 400 miles of electric range with access to fast charging at a rate of $400 \mathrm{~kW}$. The default charging behavior is "home-dominant," meaning that drivers have access to and prefer to charge at home (or the vehicle depot when applicable) and with public charging used to fill gaps during the day. All vehicles are modeled as having access to charging at the locations of their overnight park events (CYC fleet depot and drivers' residences).

The charging demand simulation generates a set of charging sessions required to satisfy the travel patterns displayed in the data in a way that maximizes electric miles traveled and minimizes operational cost. These charging sessions are then post-processed spatially and temporally to output electric vehicle supply equipment (EVSE) requirements and to support fleet operation. Beyond quantifying the required amount of EVSE, the analysis of the charging sessions can also inform the nature of the fleet charging demand. Load profiles describing the charging energy needed by time of day are explored further in the Modeling Results section.

Adequate EVSE support for fleet vehicles is fundamentally different than for personally owned vehicles. Fleet vehicles may be utilized by multiple fleet employees, experience higher utilization, are driven longer distances, and do not typically experience long dwell times throughout the middle of the day (such as observed with personal vehicles at workplaces). The unique operation of fleet vehicles motivated several assumptions not present in previous EVI-Pro studies addressing electrification of personal vehicles. First, it is assumed that direct current fast charging (DCFC) is the only level of charging available for public charging opportunities. Drivers utilizing fleet vehicles during the day are on-shift, and thus unlikely to be willing to charge at slower speeds during their workday. Second, due to the lack of a conventional workplace dwell opportunities, no workplace charging is considered. Finally, the CYC taxi vehicles may be parked overnight at either drivers' residences or the fleet depot. These locations are categorized as "overnight parking" where access to only L2 charging may be available.

\section{Modeling Results}

Simulations were performed using EVI-Pro and the processed CYC travel data as an input. Day-level simulations were performed for each vehicle-day, identifying the amount of charging necessary during dwell events to support vehicles modeled as BEV250s. Vehicle constraints (battery size, charge power), travel constraints (dwell frequency, dwell time), and infrastructure constraints (charging availability) are all considered when evaluating charging solutions. Note that the combination of factors influencing charging outcomes may result in no successful charging solution for a portion of the simulated vehicle-days; these "unserved" vehicles typically travel large portions of the day, resulting in high mileage and limited charging opportunities. Use of the present-day taxi operation implicitly assumes that future drivers will attempt to utilize the BEV250s in a manner consistent with current taxi operation. It is assumed that drivers of the "unserved" vehicles would be required to alter their travel behavior and seek charging opportunities.

The feasibility of fleet electrification is also a function of the vehicle specifications. An additional section is included that explores fleet performance for varying vehicle capabilities, including low range electric vehicles with modest charging powers, electric vehicles with similar capabilities to currently available models (BEV250), and potential future electric vehicles with greater range and charge acceptance.

\section{Scenarios with Varying Infrastructure Access}

Successful electrification of a fleet of vehicles can be accomplished through a variety of strategies. Charging requirements necessary to support CYC fleet operation were evaluated for two specific infrastructure scenarios with differing levels of charging availability. For each scenario, BEV250 vehicles with a maximum charge power of $50 \mathrm{~kW}$ are modeled as the fleet vehicle. Results for each scenario are discussed in the subsequent section; primary outputs include charge events, unserved vehicle counts, and analysis of fleet-wide load profiles.

\section{Public Charging, Residential Charging, and Depot Charging}

The first scenario assumes generous access to electric vehicle charging. Fleet vehicles have access to charge at publicly accessible stations, at the fleet depot, and at home. Overnight charger access is prescribed at drivers' residencies and the CYC depot while the locations of public chargers are generated from the spatial and temporal analysis within EVI-Pro.

\section{Public Charging Only}

The second scenario assumes no charging investment by the fleet, leaving only public charging as the sole opportunity for vehicle 
charging. Results of this scenario will inform the amount of public charging access needed and whether overnight charging is necessary to support fleet operation.

\section{Infrastructure Sensitivity Results}

The 35,112 vehicle-days within the 13-month CYC data were input into EVI-Pro along with infrastructure assumptions for Scenario 1 and Scenario 2. Key results from the two simulations can be found in Table 1. Most notable is the vast difference in served vehicle-days. Access to overnight charging and publicly available DCFC adequately services $90.5 \%$ of CYC vehicle-days, a number that drops to only $40 \%$ when overnight charging is no longer available. Additionally, DCFC participation rises from 0.17 events per vehicleday when overnight charging is available to $1.63 \mathrm{DCFC}$ events per vehicle-day when fast charging is the only option for replenishing battery energy.

Table 1. Selected EVI-Pro Results for Scenario 1 and Scenario 2 for analysis of 35,112 CYC vehicle-days simulated as being driven by BEV250's

\begin{tabular}{|c|c|c|c|c|}
\hline & $\begin{array}{c}\text { Percent Vehicle- } \\
\text { Days with }>0 \\
\text { DCFC Events }\end{array}$ & $\begin{array}{c}\text { Vehicle-Days } \\
\text { With DCFC } \\
\text { Events }\end{array}$ & $\begin{array}{c}\text { Served } \\
\text { Vehicle-Days } \\
{[\% \text { of Total }]}\end{array}$ & $\begin{array}{c}\text { DCFC } \\
\text { events per } \\
\text { vehicle-day }\end{array}$ \\
\hline $\begin{array}{c}\text { Infrastructure } \\
\text { Scenario 1 }\end{array}$ & $8.66 \%$ & 5655 & $\begin{array}{c}33604 \\
{[\mathbf{9 5 . 7 \% ]}}\end{array}$ & 0.13 \\
\hline $\begin{array}{c}\text { Infrastructure } \\
\text { Scenario 2 }\end{array}$ & $100.0 \%$ & 22312 & $\begin{array}{c}14001 \\
{[\mathbf{3 9 . 9 \%}]}\end{array}$ & 1.59 \\
\hline
\end{tabular}

EVI-Pro also outputs spatial information associated with charging events. The unique DCFC events in Table 1 for both scenarios are shown spatially throughout the Columbus region in Figures 11 and 12. Note that the number of charge event clusters does not rise proportionately to the number of unique events; a single cluster may be comprised of multiple charge events (signified by color and transparency). Additionally, the charging heatmap only shows the charging sessions of served vehicles. Many vehicles participating in charge events represented in Figure 12 are unserved due to the limited infrastructure availability in Scenario 2. Accurately depicting infrastructure requirements for both scenarios may thus require additional analysis capturing charging requirements for unserved vehicles. Additional discussion may be found in the Future Work section.

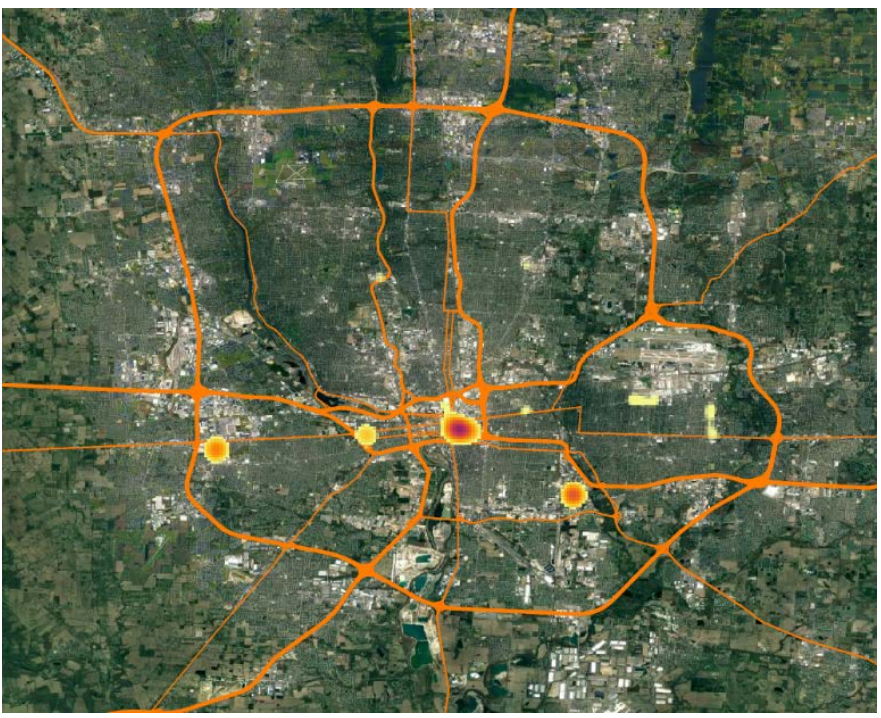

Figure 11. Heatmap of DCFC charging locations assuming access to overnight charging.

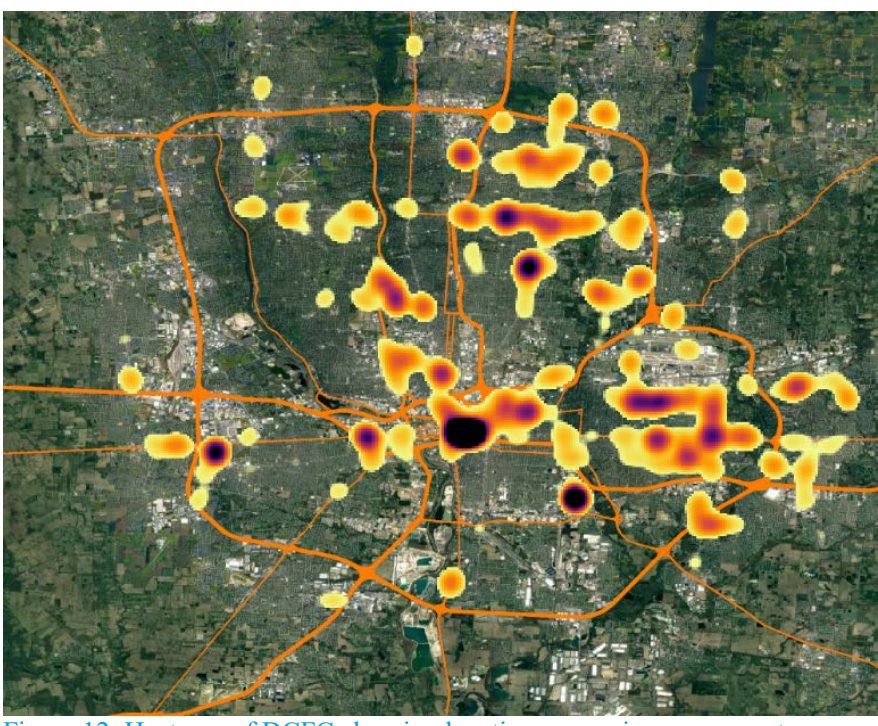

Figure 12. Heatmap of DCFC charging locations assuming no access to overnight charging.

\section{Infrastructure Sensitivity Charging Profiles}

While the primary output of EVI-Pro simulations are estimates for consumer charging infrastructure requirements, the highly resolved nature of EVI-Pro simulations lends itself well to estimating aggregate charging load profiles for the vehicle fleet. This section presents simulated load profiles by time of day and location type from the charging sessions of served vehicles from Scenario 1 and Scenario 2 on weekdays. Weekdays are emphasized given the larger number of miles driven by $\mathrm{CYC}$ vehicles and increased charging requirements.

Figures 13 and 14 show weekday load profiles presented on a pervehicle basis. Both figures are overlaid with the vehicle trip frequency trace introduced in the Columbus Yellow Cab Data section to provide context for when vehicles are driving and when they are charging. For Scenario 1, overnight charging presents most of the energy delivered to fleet vehicles with DCFC supplying additional power primarily during the day. The charging peak of $6.18 \mathrm{~kW} /$ vehicle for Scenario 1 occurs at approximately $9 \mathrm{PM}$, tapering off as trip frequency rises during the subsequent morning. Beyond supplying more convenient energy, access to overnight charging is also shown to minimize the amount of required unique charge events per day.

Meanwhile, drivers in Scenario 2 are shown to receive most of their energy during peak hours of fleet operation. The lack of available overnight opportunities forces fleet vehicles to be fully reliant on the public infrastructure. Inspection of Figure 14 illustrates that the peak power $6.20 \mathrm{~kW} /$ vehicle is only slightly higher in Scenario 2; the small difference in power, despite the larger amount of high-power DCFC events, is likely explained by the different number of served vehicles between Scenarios 1 and 2. Many high-mileage vehicle-days with correspondingly high charging requirements are present within Scenario 1 results but absent from Scenario 2. 


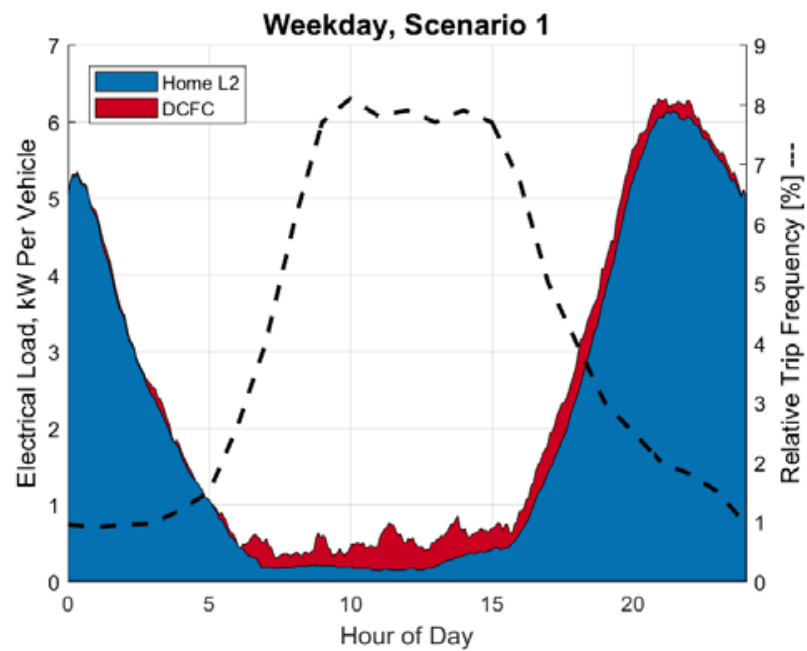

Figure 13. Per-vehicle electrical load demanded by fleet vehicles with available access to overnight charging.

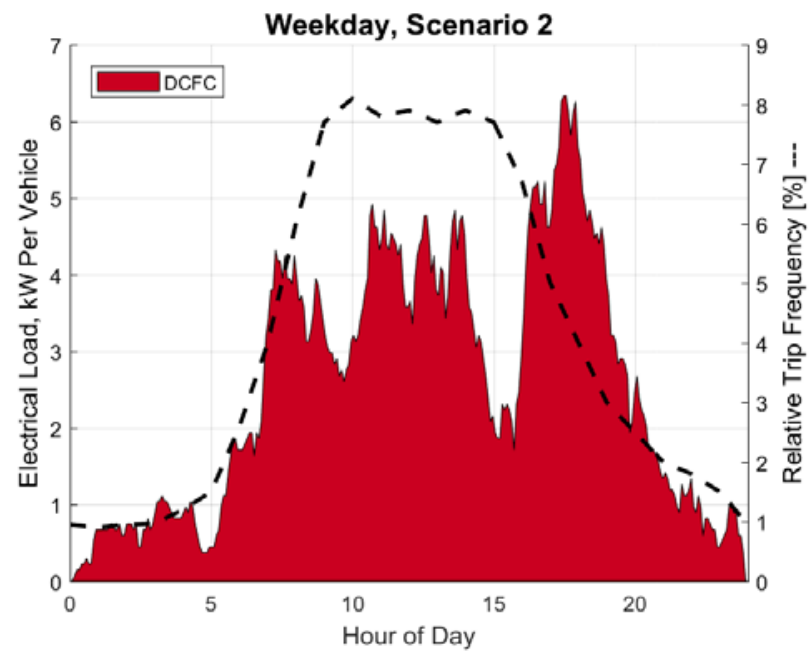

Figure 14. Per-vehicle electrical load demanded by fleet vehicles with no available access to overnight charging.

\section{Scenarios with Varying Vehicle Capabilities}

Additional analysis was performed to understand the relationship between vehicle specifications and the feasibility of CYC fleet electrification. The variable of infrastructure access, explored in the previous section, is held constant; overnight charging is assumed available for all vehicle scenarios given the favorable results for the vehicle fleet (more served vehicle-days, fewer charge events during the day, etc.). Vehicle parameters were varied from a low mileage electric vehicle to a futuristic electric vehicle with a large range and high charge acceptance. Specific parameters for each scenario are listed below with modeling results following thereafter. The parameters prescribed for Vehicle Scenario 2 are identical to the vehicle parameters assumed for infrastructure sensitivity simulations.

\section{BEV100, 50-kW Charging}

\section{BEV250, 50-kW Charging}

\section{BEV400, 400kW Charging}

The 35,112 vehicle-days were again simulated for each of the defined vehicle scenarios. In each scenario, the entire fleet was assumed to have vehicle specifications matching those above. The authors acknowledge that a real-world fleet may comprise vehicles with varying makes, models, and capabilities. Key results from the vehicle scenarios may be found in Table 2. Note that the results for Vehicle Scenario 2 are identical to those for Infrastructure Scenario 1assumptions regarding infrastructure access and vehicle capabilities are the same for these two cases.

Table 2. Selected EVI-Pro Results for Vehicle Scenarios 1, 2, and 3 for analysis of 35,112 CYC vehicle-days simulated as having access to overnight charging

\begin{tabular}{|c|c|c|c|c|}
\hline & $\begin{array}{c}\text { Percent Vehicle- } \\
\text { Days with }>0 \\
\text { DCFC Events }\end{array}$ & $\begin{array}{c}\text { Vehicle-Days } \\
\text { With DCFC } \\
\text { Events }\end{array}$ & $\begin{array}{c}\text { Served } \\
\text { Vehicle-Days } \\
\text { [\% of Total] }\end{array}$ & $\begin{array}{c}\text { DCFC } \\
\text { events per } \\
\text { vehicle-day }\end{array}$ \\
\hline $\begin{array}{c}\text { Vehicle } \\
\text { Scenario 1 }\end{array}$ & $64.78 \%$ & 7826 & $\begin{array}{c}12081 \\
{[\mathbf{3 4 . 4 \%}]}\end{array}$ & 1.21 \\
\hline $\begin{array}{c}\text { Vehicle } \\
\text { Scenario 2 }\end{array}$ & $8.66 \%$ & 4325 & $\begin{array}{c}33604 \\
{[\mathbf{9 5 . 7 \%}]}\end{array}$ & 0.13 \\
\hline $\begin{array}{c}\text { Vehicle } \\
\text { Scenario 3 }\end{array}$ & $4.88 \%$ & 1974 & $\begin{array}{c}34948 \\
{[\mathbf{9 9 . 6 \%}]}\end{array}$ & 0.06 \\
\hline
\end{tabular}

Results from the vehicle scenarios confirm that fleet vehicle capabilities are also a major variable surrounding successful fleet electrification. For instance, even when generous access to charging infrastructure is assumed (overnight charging, public DCFC), the low mileage BEVs from Vehicle Scenario 1 are only able to complete $34.4 \%$ of fleet vehicle-days. Increasing the vehicle range by 150 miles, from 100 to 250 , raises the served vehicle-day percentage to $95.7 \%$. However, increasing the range by another 150 miles (as well as the DCFC power), from 250 to 400 , raises the served vehicle-day percentage to $99.6 \%$. These numbers indicate that there may be diminishing returns surrounding fleet operation and vehicle capabilities. Economical operation may consist of a fleet primarily composed of electric vehicles with moderate specifications (Vehicle Scenario 2), with a small portion of the fleet comprised of vehicles more capable of high mileage vehicle-days (Vehicle Scenario 3, hybrid vehicles, etc.).

\section{Summary and Conclusions}

There is significant interest in electrifying light-duty commercial vehicles - both by municipalities concerned with meeting emissions targets and fleet owners concerned with lowering their operating costs. This report analyzed the feasibility of converting the Columbus Yellow Cab taxi fleet from gasoline fueled vehicles to electric vehicles through processing real-world of global positioning system data and an EVI-Pro infrastructure analysis.

Over 70 million global positioning system points were processed, representing 35,112 vehicle-days of fleet operation over a 13-month time span. Analysis revealed that a typical Columbus Yellow Cab vehicle travels 154 miles per day and operates for 280 days per year. A comparative study was performed against two datasets to understand how Columbus Yellow Cab travel behavior relates to personally owned light duty vehicles in Ohio and vehicles operated by full-time drivers working for the transportation network company RideAustin. The results revealed that Columbus Yellow Cab drivers travel significantly more miles when compared to both personal travel and TNC travel; the average Columbus Yellow Cab vehicle travels 40,000 miles per year compared to 29,000 for full-time RideAustin drivers and 11,488 for personally owned vehicles.

EVI-Pro simulations were performed for two infrastructure scenarios with differing access to overnight charging and three vehicle scenarios with differing vehicle capabilities. Simulations assumed 
that the Columbus Yellow Cab trips - originally taken by gasoline fueled vehicles - were instead taken by battery electric vehicles with prescribed vehicle ranges and levels of maximum charge power. Results confirmed that the feasibility of fleet electrification is strongly correlated to both charring infrastructure access and vehicle capabilities. For instance, successful charging solutions were identified for $95.7 \%$ of vehicle-days for Infrastructure Scenario 1, but only $39.9 \%$ of vehicle-days for Infrastructure Scenario 2. Results shown for the served vehicles indicate that overnight charging access significantly reduces DCFC activity, from 1.63 events per vehicleday to 0.17 events per vehicle-day. Additionally, load profile comparisons reveal that overnight charging provides the additional benefit of allowing drivers to replenish their vehicles primarily offshift. The reduced number of mid-day charging sessions and minimal reliance on the public infrastructure both support the importance of overnight charging. Additional analysis surrounding vehicle capabilities also revealed electric vehicle range and charging power to be correlated with successful fleet operation. Served vehicle-day percentages varied significantly between scenarios, ranging from $34.4 \%$ assuming limited vehicle specifications to $99.6 \%$ assuming futuristic vehicle specifications. The vehicle analysis also revealed potential diminishing returns associated with increasing vehicle capabilities and successful operation.

For all scenarios evaluated, a non-trivial number of Columbus Yellow Cab vehicle-days could not be supported due to large charging requirements and minimal charging opportunities. Increasing the number of served vehicle-days to $100 \%$ would require either increasing vehicle capabilities (larger charge power, larger battery size) or introducing more dwell events-drivers suspending service and seeking out charge events. Thus, given the limited availability of economical, high mileage electric vehicles beyond 250 miles, full taxi electrification may require adjustments in operation even with generous access to charging. An additional solution could be partial adoption of electric vehicles for low to moderate mileage travel-days with the remaining portion of the fleet capable of fueling on gasoline serving drivers with high mileage travel-days.

\section{Future Research}

CYC's interest in electrification has already resulted in the acquisition of multiple BEVs with specifications similar to the BEV250s modeled in this report. The authors are interested in comparing the real-world behavior of electric taxi drivers to the modeled behavior in EVI-Pro. Key areas of future research potentially include studying any changes in driver dVMT, BEV taxi operation compared to privately owned BEVs, and any changes in driver behavior.

The authors are also interested in exploring expansions to EVI-Pro to more accurately accommodate high mileage travel-days. Unserved travel days have not been prominent in previous infrastructure studies exploring travel data for personally owned vehicles, which tend to consist of lower mileage vehicle-days. Assumptions of no change in driver behavior between gasoline operation and electric operation proved limiting for some aspects of this study. Future research may provide a means for incorporating charging solutions / behavior modifications to increase the number of served vehicles and facilitate more direct comparisons between different scenarios.

Finally, comparisons between travel behavior for privately owned vehicles, taxi vehicles, and vehicles dispatched by TNCs warrant further exploration. The results shown within this paper are limited in scope given the presence of data from only a single taxi fleet and only a single TNC company which was located in a separate region. Future work may expand upon these comparisons as more taxi and
TNC data becomes available, and as mode choice behavior for personal travel evolves.

\section{References}

1. "Fast Facts U.S. Transportation Sector Greenhouse Gas Emissions 1990 - 2016," Office of Transportation and Air Quality, July 2018, https://nepis.epa.gov/Exe/ZyPDF.cgi?Dockey=P100USI5.pdf

2. California State Legislature. Cooper (A): AB-615 Air Quality Improvement Program: Clean Vehicle Rebate Project (California, 2017), http://leginfo.legislature.ca.gov/faces/billNavClient.xhtml?bill i $\mathrm{d}=201720180 \mathrm{AB} 615$

3. Governor’s Press Office, “Governor Cuomo Announces $\$ 250$ Million Initiative to Expand Electric Vehicle Infrastructure Across New York State," May 2018,

https://www.governor.ny.gov/news/governor-cuomo-announces250-million-initiative-expand-electric-vehicle-infrastructureacross

4. California Public Utilities Commission, "CPUC Approval of Utility Projects Keeps California at Forefront of Transportation Electrification," May 2018, http://docs.cpuc.ca.gov/PublishedDocs/Published/G000/M215/K 467/215467739.PDF

5. Wenzel, T., Rames, C., Kontou, E., Henao, A., "Travel and Energy Implications of Ridesourcing Service in Austin, Texas," Forthcoming.

6. Tefft, B. C., “American Driving Survey: 2015- 2016," AAA Foundation for Traffic Safety, January 2018, http://aaafoundation.org/wp-content/uploads/2018/02/180019 AAAFTS-ADS-Research-Brief.pdf

7. California State Legislature. Skinner (S): SB-1014 California Clean Miles Standard and Incentive Program: zero-emission vehicles (California 2018),

https://leginfo.legislature.ca.gov/faces/billCompareClient.xhtml? bill id=201720180SB1014

8. Nelson, L., "Uber and Lyft Have Devastated L.A.'s Taxi Industry, City Records Show," Los Angeles Times, April 2016, http://www.latimes.com/local/lanow/la-me-ln-uber-lyft-taxis-la20160413-story.html

9. City Council of the City of Austin. An Ordinance Amending City Code Chapter 13-2 (Ground Transportation Passenger Services) Related to Regulation of Taxicabs and Limousines, and Repealing Certain Requirements Related to Taxicabs and Taxicab Franchises (Texas 2018), http://www.austintexas.gov/edims/document.cfm?id=301222

10. New York City Taxi and Limousine Commission, "New York Taxi and Limousine Commission Resolution Approving Pilot Program to Allow Licensed E-Hail Apps to Offer Flexible Fares in Taxicabs," March 2018, http://www.nyc.gov/html/tlc/downloads/pdf/flex_fare_resolution 03 29.pdf

11. Wood, E., Rames, C., Muratori, M., Raghaven S., Young, S., "Charging Electric Vehicles in Smart Cities: An EVI-Pro Analysis of Columbus, Ohio," NREL/TP-5400-70367, February 2018, https://www.nrel.gov/docs/fy18osti/70367.pdf

12. Wood, E., Rames, C., Muratori, M., Raghavan, S. et al., "National Plug-in Electric Vehicle Infrastructure Analysis," Report from the US DOE Office of Energy Efficiency and Renewable Energy, September 2017, https://www.nrel.gov/docs/fy17osti/69031.pdf

13. Wood, E., Raghavan, S., Rames, C., Eichman, J. et al., "Regional Charging Infrastructure for Plug-in Electric Vehicles: A Case Study of Massachusetts," NREL/TP-5400- 67436, Jan 2017, https://www.nrel.gov/docs/fy17osti/67436.pdf 


\section{Contact Information}

Matt Moniot matthew.moniot@nrel.gov

National Renewable Energy Laboratory

15013 Denver West Pkwy MS 1634

Golden, CO 80401

\section{Acknowledgments}

This report and the work described were sponsored by the U.S.

Department of Energy (DOE) Vehicle Technologies Office (VTO) under the Systems and Modeling for Accelerated Research in Transportation (SMART) Mobility Laboratory Consortium, an initiative of the Energy Efficient Mobility Systems (EEMS) Program. The authors acknowledge John Smart of Idaho National Laboratory for leading the Advanced Fueling Infrastructure Pillar of the SMART Mobility Laboratory Consortium. The following DOE Office of Energy Efficiency and Renewable Energy (EERE) managers played important roles in establishing the project concept, advancing implementation, and providing ongoing guidance: David Anderson, and Erin Boyd. Finally, the authors would like to also thank Morgan Kauffman of Columbus Yellow Cab for his on-going collaboration.

The U.S. Government retains and the publisher, by accepting the article for publication, acknowledges that the U.S. Government retains a nonexclusive, paid-up, irrevocable, worldwide license to publish or reproduce the published form of this work, or allow others to do so, for U.S. Government purposes.

\section{Definitions/Abbreviations}

BEV

BEV250

CYC

DCFC

dVMT

EVI-Pro

EVSE

GHG

GPS

NREL

PEV

TNC battery electric vehicle

battery electric vehicle with 250 miles of range

Columbus Yellow Cab

direct current fast charging

daily vehicle miles traveled

Electric Vehicle Infrastructure Projection Tool

electric vehicle supply equipment

greenhouse gas emission

global positioning system

National Renewable Energy

Laboratory

plug-in electric vehicle

transportation network company 